INPLASY

PROTOCOL

To cite: Sun et al. Antibiotic treatment time for Gramnegative bacilli bloodstream infection: a systematic review and meta analysis. Inplasy protocol 202130057. doi: 10.37766/inplasy2021.3.0057

Received: 16 March 2021

Published: 16 March 2021

Corresponding author: Limin Sun

sunxiaominwater@163.com

Author Affiliation:

The first affiliated hospital of zhengzhou university

Support: None.

Review Stage at time of this submission: The review has not yet started.

Conflicts of interest: None declared.

\section{Antibiotic treatment time for Gram- negative bacilli bloodstream infection: a systematic review and meta analysis}

\author{
Sun, L1; Yang, Z².
}

Review question / Objective: P: Patients with Gram-negative bacteria bloodstream infection (regardless of source) I: The duration of antibiotic treatment is divided into short-term treatment group ( $\leq 7$ days) and long-term treatment group ( $>7$ days). O: Short-term and long-term mortality, recurrence rate of bloodstream infection, incidence of drug-resistant bacteria. S: Observational study. Objective: The aim of the present study is to evaluate short versus longer courses of antibiotic treatment for bloodstream infection due to Gram-negative bacilli in clinical outcomes.

Information sources: We will search the target study from MEDLINE, Embase, CENTRAL, the Web of Science, and reviews of grey literature on Open Grey. There is no restriction will be imposed on the data of publication, and all studies published before March 2021 will be included. And no language restrictions on retrieval at the same time.

INPLASY registration number: This protocol was registered with the International Platform of Registered Systematic Review and Meta-Analysis Protocols (INPLASY) on 16 March 2021 and was last updated on 16 March 2021 (registration number INPLASY202130057).

\section{INTRODUCTION}

Review question / Objective: P: Patients with Gram-negative bacteria bloodstream infection (regardless of source) I: The duration of antibiotic treatment is divided into short-term treatment group ( $\leq 7$ days) and long-term treatment group ( $>7$ days). 0 : Short-term and long-term mortality, recurrence rate of bloodstream infection, incidence of drug-resistant bacteria. S: Observational study. Objective: The aim of 
the present study is to evaluate short versus longer courses of antibiotic treatment for bloodstream infection due to Gram-negative bacilli in clinical outcomes.

Condition being studied: The current guidelines of the American Society of Infectious Diseases recommend that the treatment time for intravascular catheterrelated gram-negative bacteremia should be 7 to 14 days, but there is no consensus on the best time for antimicrobial treatment of non-catheter-related gram-negative bacteremia. A recently published study on Gram-negative bacteria bloodstream infection compares the clinical results of short-term and long-term treatment in an attempt to determine the best treatment time, but their results are controversial. So we are going to conduct a meta analysis and systematic review to sort it out.

\section{METHODS}

Search strategy: (( ( "Duration of Therapy"[Mesh]) OR $(((()((()(($ Week[Title) Abstract]) OR (Day[Title/Abstract])) OR (Long[Title/Abstract])) OR (Short[Title/ Abstract])) OR (Duration of Therapy[Title/ Abstract])) OR (Duration of antibiotic treatment [Title/Abstract])) OR (Duration[Title/Abstract])) OR (Hour[Title/ Abstract])) OR (Time[Title/Abstract])) OR (Therapy Duration[Title/Abstract])) OR (Duration of Treatment[Title/Abstract])) OR (Treatment Duration[Title/Abstract])) OR (month[Title/Abstract]))) AND

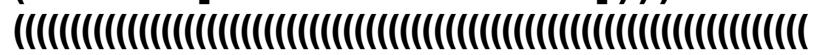
)(()(()(()(()Agents, Anti-Bacterial[Title) Abstract]) OR (Anti Bacterial Agents[Title/ Abstract])) OR (Antibacterial Agents[Title/ Abstract])) OR (Agents, Antibacterial[Title/ Abstract])) OR (Antibacterial Agent[Title/ Abstract])) OR (Agent, Antibacterial[Title/ Abstract])) OR (Anti-Bacterial Compounds[Title/Abstract])) OR (Anti Bacterial Compounds[Title/Abstract])) OR (Compounds, Anti-Bacterial[Title/ Abstract])) OR (Anti-Bacterial Agent[Title/ Abstract])) OR (Agent, Anti-Bacterial[Title/ Abstract])) OR (Anti Bacterial Agent[Title/ Abstract])) OR (Anti-Bacterial Compound[Title/Abstract])) OR (Anti Bacterial Compound[Title/Abstract])) OR
(Bacteriocidal Agents[Title/Abstract])) OR (Agents, Bacteriocidal[Title/Abstract])) OR (Bacteriocidal Agent[Title/Abstract])) OR (Bacteriocide[Title/Abstract])) OR (Bacteriocides[Title/Abstract])) OR (AntiMycobacterial Agents[Title/Abstract])) OR (Anti Mycobacterial Agents[Title/Abstract])) OR (Anti Mycobacterial Agent[Title/ Abstract])) OR (Anti Mycobacterial Agent [ Title / Abstract])) OR (Antimycobacterial Agent[Title/Abstract])) OR (Antibiotics[Title/Abstract])) OR (Antibiotic[Title/Abstract])) OR (Inhibitors, beta-Lactamase[Title/Abstract])) OR (beta Lactamase Inhibitor[Title/Abstract])) OR (Inhibitor, beta Lactamase[Title/Abstract])) OR (Lactamase Inhibitor, beta[Title/ Abstract])) OR (beta Lactamase Inhibitors[Title/Abstract])) OR (beta Lactamase Antagonists[Title/Abstract])) OR (beta-Lactamase Inhibitor[Title/Abstract])) OR (Carbapenem Antibiotics[Title) Abstract])) OR (Carbapenem[Title/ Abstract])) OR (Merrem[Title/Abstract])) OR (Ronem[Title/Abstract])) OR (Penem[Title/ Abstract])) OR (SM 7338[Title/Abstract])) OR (Imipemide[Title/Abstract])) OR (Imipenem, Anhydrous[Title/Abstract])) OR (Anhydrous Imipenem[Title/Abstract])) OR (Imipenem Anhydrous[Title/Abstract])) OR (MK-0787[Title/Abstract])) OR (Polymyxin[Title/Abstract])) OR (Polymyxin M[Title/Abstract])) OR (Polymyxin E[Title/ Abstract])) OR (Colimycin[Title/Abstract])) OR (Coly-Mycin[Title/Abstract])) OR (Colistin Sulfate[Title/Abstract])) OR (Polymyxin B Sulfate[Title/Abstract])) OR (Aerosporin[Title/Abstract])) OR (Tegacycline[Title/Abstract])) OR (Piperacillin-Tazobactam Combination Product[Title/Abstract])) OR (Piperacillin Tazobactam Combination Product[Title/ Abstract])) OR (Piperacillin Tazobactam [Title/Abstract])) OR (Tazocin [Title/Abstract])) OR (Tazocillin[Title/Abstract])) OR (Tazocel[ Title/Abstract])) OR (Pipercillin[Title/Abstract])) OR (Piperacillin Sodium[Title/Abstract])) OR (Piperacillin Monosodium Salt[Title/Abstract])) OR (T1220[Title/Abstract])) OR (Piperacillin Ratiopharm[Title/Abstract])) OR (Pipera Hameln [Title/Abstract])) OR (AB Piperacillin[Title/Abstract])) OR (Piperacillin 
Curasan [Title/Abstract])) OR (Aminoglycoside[Title/Abstract])) OR (Fortum[Title/Abstract])) OR (Fortaz[Title/ Abstract])) OR (Ceftazidime Pentahydrate[Title/Abstract])) OR (Tazidime[Title/Abstract])) OR (Ceftazidime Anhydrous[Title/Abstract])) OR (beta Lactams [Title/Abstract])) OR (betaLactam[Title/Abstract])) OR (beta Lactam [Title/Abstract])) OR (Cefoperazon [Title/Abstract])) OR (Cefoperazone Sodium[Title/Abstract])) OR (Cefobid[Title/Abstract])) OR (Cefdinir[Title/ Abstract])) OR (Cephalexin[Title/Abstract])) OR (Cephaloridine[Title/Abstract])) OR (Cefuroxime[Title/Abstract])) OR (Cefepime[Title/Abstract])) OR (Cefazolin[Title/Abstract])) OR

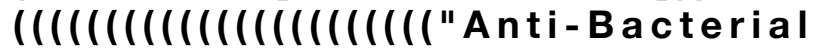
Agents"[Mesh]) OR "beta-Lactamase Inhibitors "[Mesh]) OR "Cephalosporins"[Mesh]) OR "Carbapenems" [Mesh]) OR

"Meropenem" [Mesh]) OR

"I m ipenem" [M esh ]) OR "Polymyxins"[Mesh]) OR "Polymyxin B"[Mesh]) OR "Colistin"[Mesh]) OR "Piperacillin"[Mesh]).

Participant or population: Patients with Gram-negative bacteria bloodstream infection.

Intervention: Short-term treatment group ( $\leq 7$ days).

Comparator: Long-term treatment group ( $>7$ days).

Study designs to be included: Observational study.

Eligibility criteria: Inclusion criteria: 1. Patients with gram-negative bloodstream infection; 2. The age is not less than 18 years old; 3 . The clinical data were complete.

Information sources: We will search the target study from MEDLINE, Embase, CENTRAL, the Web of Science, and reviews of grey literature on Open Grey. There is no restriction will be imposed on the data of publication, and all studies published before March 2021 will be included. And no language restrictions on retrieval at the same time.

Main outcome(s): Short-term and long-term mortality, recurrence rate of bloodstream infection, incidence of drug-resistant bacteria.

Additional outcome(s): None.

Data management: Short-term and longterm mortality, recurrence rate of bloodstream infection, incidence of drugresistant bacteria.

Quality assessment / Risk of bias analysis: The literature quality evaluation method adopts the Newcastle-Ottawa scale score evaluation form, which is evaluated independently by two participants. If the conclusions are inconsistent, the final decision will be made by the third-party experts.

Strategy of data synthesis: If there are at least 4 studies that can be used for data consolidation, meta analysis is used. If the number of studies is less than 4, a systematic review will be conducted.

Subgroup analysis: We will conduct a subgroup analysis, including trial types, patient types, pathogen source, types of antibiotics, duration of application of antibiotics, and different methods for determining clinical outcomes.

Sensitivity analysis: We will evaluate the stability of the meta analysis results at the end, that is, conduct a sensitivity analysis. It includes changing the criteria for selecting literature (conference abstracts or articles), choosing non-English articles or not, changing different observation outcomes, and changing statistical models. Then re-conduct the meta analysis to see if the decision of each step will affect the result.

Language: There are no language restrictions. 
Country(ies) involved: All the authors are from China.

Other relevant information: None.

Keywords: Enterobacteriaceae, Gram negative, antibiotic treatment, antimicrobial therapy, bacteremia, bloodstream infection, optimal duration, sepsis, septicemia.

Contributions of each author:

Author 1 - Limin Sun - Raise clinical questions and design this meta-analysis. Formulate the retrieval strategy, find the relevant literature, and determine the inclusion and exclusion criteria of the literature. Search the literature and screen the required literature. Evaluate the quality of the research. Extract data. Meta analysis was performed. Write the article.

Email: sunxiaominwater@163.com

Author 2 - Ziyue Yang - Search the literature and screen the required literature. Evaluate the quality of the research. Extract data. Meta analysis was performed. Write the article.

Email: yzytuki@163.com 